\title{
Use of a Tunnelled Pleural Catheter for the Management of Refractory Hepatic Hydrothorax: A New Therapeutic Option
}

\author{
Pascale Mercky ${ }^{\mathrm{a}} \quad$ Lama Sakr $^{\mathrm{b}} \quad$ Laurent Heyries $^{\mathrm{a}} \quad$ Xavier Lagrange $^{\mathrm{a}} \quad$ José Sahel $^{\mathrm{a}}$ \\ Hervé Dutau ${ }^{b}$

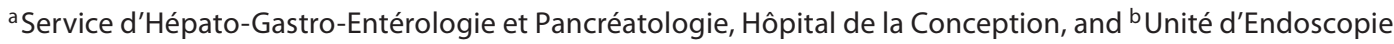 \\ Thoracique, Hôpital Sainte-Marguerite, Marseille, France
}

\section{Established Facts}

- Small-bore indwelling pleural catheters $\left(\right.$ PleurX $\left.{ }^{\circledR}\right)$ provide effective symptom improvement in up to $95 \%$ of patients with malignant pleural effusion.

- Long-lasting spontaneous pleurodesis has been achieved in almost half of the patients who underwent (PleurX) insertion for management of malignant pleural effusion.

\section{Novel Insights}

- The use of small-bore indwelling pleural catheters $\left(\right.$ PleurX $\left.{ }^{\circledR}\right)$ for the management of refractory benign pleural effusions has only been rarely reported in the recent literature.

- This is the first case report describing effective management of hepatic hydrothorax in a patient with advanced cirrhosis, using PleurX catheter. Spontaneous pleurodesis was achieved without recurrent pleural effusion at a 6-month follow-up.

\section{Key Words}

Hepatic hydrothorax - Pleural effusion - Pleural transudate Pleur $X^{\circledR} \cdot$ Pleural catheter

\begin{abstract}
Refractory hepatic hydrothorax poses a challenging therapeutic dilemma, as treatment options are limited. Herein, we describe the case of a 48-year-old lady with advanced cirrhosis and recurrent transudative pleural effusion despite a sodium-restricted diet, optimal diuretic therapy and trans-
\end{abstract}

jugular intrahepatic portosystemic shunt. Given the patient's platelet and coagulation disorders, thoracoscopic pleurodesis was deemed unsafe. Instead, a tunneled pleural catheter $\left(\right.$ Pleur $X^{\circledR}$ ) was inserted under local anesthesia. Pleural drainage was achieved at the time of catheter placement and subsequently according to the patient's symptoms. Symptomatic improvement and gradual decrease of drainage volumes were noted. Six months following placement of PleurX, methicillin-resistant Staphylococcus aureus cellulitis at the insertion site prompted catheter removal. No pleural effusion was seen on chest X-ray at that time. Subsequent

\section{KARGER}

Fax +41613061234 E-Mail karger@karger.ch www.karger.com (c) 2010 S. Karger AG, Basel

0025-7931/10/0804-0348\$26.00/0

Accessible online at:

www.karger.com/res
Dr. Hervé Dutau

Thoracic Endoscopy Unit

Hôpital Sainte-Marguerite, 270, Bd de Sainte-Marguerite

FR-13274 Marseille Cedex 09 (France)

Tel. +33 4917446 94, Fax +33 4917454 24, E-Mail herve.dutau@ mail.ap-hm.fr 
follow-up revealed spontaneous pleurodesis, as no recurrence of pleural effusion was seen over a 6-month follow-up period. Very few data are available with regard to the use of indwelling pleural catheters for benign transudative pleural effusion, and more specifically hepatic hydrothorax. Herein, we present this novel potential indication of the indwelling pleural catheter and illustrate the successful clinical outcome.

Copyright ๑ 2010 S. Karger AG, Basel

\section{Introduction}

Recent introduction of PleurX ${ }^{\circledR}$-tunneled pleural catheters to the therapeutic armamentarium of malignant pleural effusion (MPE) has gained wide acceptance as an alternative to other palliative options, when the latter are deemed nonsuccessful or sometimes contraindicated. These small-bore indwelling pleural catheters have provided effective outpatient management of MPE, by allowing symptomatic relief of respiratory symptoms, and achieving spontaneous pleurodesis in almost half of the eligible patients [1]. However, their use in chronic refractory benign pleural effusions has only rarely been reported in the recent literature. We report herein the case of refractory hepatic hydrothorax successfully managed with a PleurX-tunneled catheter in a patient with advanced cirrhosis.

\section{Case Report}

A 48-year-old female was admitted to hospital because of diuretic-refractory ascites and right-sided pleural effusion. She had a 1-month history of worsening dyspnea associated with increasing abdominal girth. She had both a history of alcohol abuse $(90 \mathrm{~g}$ of alcohol/day) and hepatitis $\mathrm{C}$, and was diagnosed with cirrhosis following ascitic decompensation in 2006. Ascites had been well controlled since then on a treatment regimen consisting of a lowsodium diet, a combination of diuretics (furosemide $20 \mathrm{mg} / \mathrm{day}$ and spironolactone $50 \mathrm{mg} /$ day) and strict alcohol restriction. No underlying risk factors for recurrent hepatic decompensation were identified on admission.

\section{Physical Examination}

Upon admission, the patient was afebrile with a pulse rate of 102 beats/min, a respiratory rate of $24 / \mathrm{min}$, and blood pressure of $100 / 70 \mathrm{~mm} \mathrm{Hg}$. Chest examination revealed dullness to percussion and reduced breath sounds over the right hemithorax. Moderate ascites and bilateral leg edema were noted.

\section{Radiological Examination}

Chest X-ray revealed near total opacification of the right hemithorax associated with contralateral mediastinal shift, raising the suspicion of a massive pleural effusion (fig. 1a).

PleurX ${ }^{\circledR}$ for the Management of

Refractory Hepatic Hydrothorax

\section{Laboratory Investigation}

The main disorders found during the laboratory investigations were platelets at $58 \times 10^{3} \mu \mathrm{l}$ and prothrombin time $26.7 \mathrm{~s}$ (normal 12-15 s). Child-Pugh and Model for End-Stage Liver Disease (MELD) scores were 13 and 18, respectively. High-volume therapeutic thoracentesis was performed, and 1.5 liter of yellowcitrine pleural fluid was removed. Pleural fluid analysis revealed a transudative effusion (protein content $15 \mathrm{~g} / \mathrm{l}$ ) without any suspicious cellular elements.

\section{Therapeutic Intervention}

Given the refractory nature of ascites and hepatic hydrothorax despite optimal medical therapy, a transjugular intrahepatic portosystemic shunt (TIPS) was undertaken. Portosystemic pressure gradient decreased from 20 to $13 \mathrm{~mm} \mathrm{Hg}$. However, persistent right pleural effusion in a symptomatic patient was noted despite TIPS patency on Doppler ultrasound examination in addition to optimal medical therapy (fig. 1b). Given the patient's severe platelet and coagulation disorders (prothrombin time of $26.7 \mathrm{~s}$, platelet count $58 \times 10^{3} \mu \mathrm{l}$ ), we opted for a noninvasive therapeutic alternative. A tunneled indwelling pleural catheter (PleurX; Denver Biomedical, Golden, Colo., USA) was inserted in a bronchoscopy suite under local anesthesia in an outpatient setting. Pleural fluid drainage was achieved at the time of catheter placement, and subsequently according to the patient's symptoms (fig. 2a). Indeed, the patient was managed by serial and repeated fluid drainage when needed (i.e. dyspnea worsening associated with a recurrence of pleural effusion on chest X-ray). Symptomatic improvement and gradual decrease in drainage volumes were noted. Six months following catheter insertion, methicillin-resistant Staphylococcus aureus cellulitis was found at the insertion site, justifying catheter removal. No evidence of pleural effusion was found on chest X-ray at that time. Six months following catheter withdrawal, there was still no evidence of recurrent effusion (fig. 2b). No recurrence of ascites was noted on subsequent follow-up due to the patient's compliance with a sodium-restricted diet, alcohol abstinence, and optimal diuretic therapy.

\section{Discussion}

Pleural effusion develops in 5-12\% of patients with end-stage cirrhotic liver disease. It is commonly seen in conjunction with ascites and is most often right-sided (65-85\% of cases). In contrast to the peritoneal cavity, the pleural space has a relatively limited compliance, and therefore even moderate amounts of pleural fluid can cause significant respiratory symptoms, including dyspnea and chest pain. Several mechanisms have been proposed to explain the pathogenesis of pleural effusion in patients with cirrhotic liver disease and portal hypertension. Two currently accepted theories consist of: (1) hypoalbuminemia and decreased intravascular colloid osmotic pressure secondary to diminished protein synthesis and (2) direct passage of ascitic fluid into the pleural space through minute diaphragmatic defects. These

Respiration 2010;80:348-352 
Fig. 1. a Chest radiograph before TIPS. b Chest radiograph 1 month following TIPS and just before PleurX insertion.
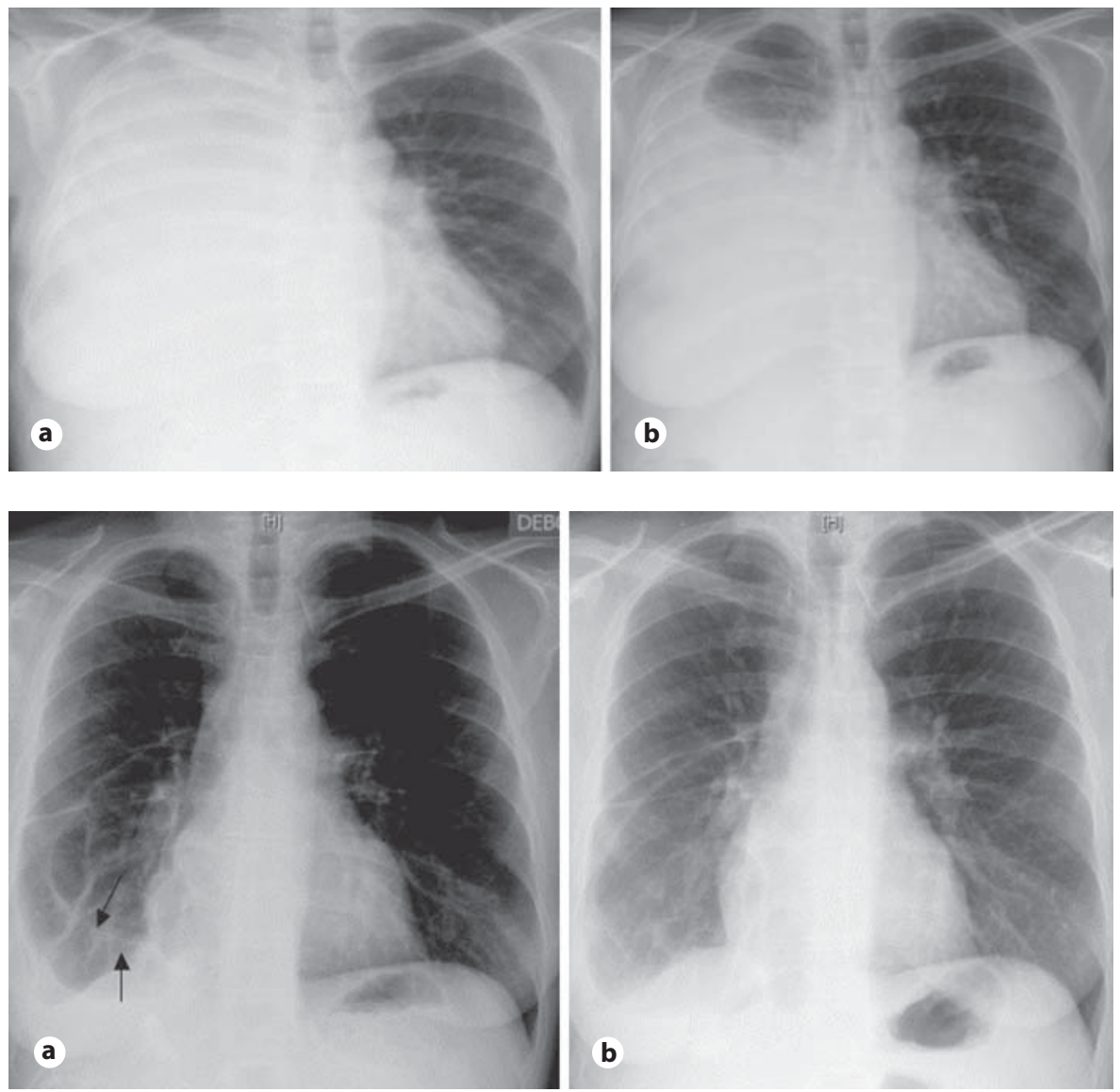

Fig. 2. a Chest radiograph 1 month following PleurX insertion (black arrows). b Chest radiograph 6 months after PleurX withdrawal. to medical therapy despite increasing doses (spironolactone up to $400 \mathrm{mg} /$ day and furosemide up to $160 \mathrm{mg} /$ day). Moreover, potential renal function impairment and the risk of electrolyte imbalance might not allow an increase in dosage. In such circumstances, more invasive therapeutic strategies should be considered [2-5].

Therapeutic thoracentesis is a safe and simple procedure, allowing rapid relief of dyspnea in patients with large and refractory effusions. However, therapeutic thoracentesis is not the optimal treatment strategy for recurrent hepatic hydrothorax, as it can lead to substantial protein depletion without preventing fluid reaccumulation $[2,3]$.

TIPS is another therapeutic modality for patients with refractory hydrothorax. It provides symptomatic relief in $70-80 \%$ of subjects $[6,7]$. However, serious complications have been encountered following this procedure, including shunt occlusion and hepatic encephalopathy in a significant proportion of patients ( 25 and $30 \%$, respectively) [8]. In our patient, hepatic hydrothorax was refractory to TIPS, warranting alternative treatment options. 
Chemical pleurodesis has been previously attempted in patients with refractory hydrothorax, using chest tube thoracostomy, conventional thoracoscopy or video-assisted thoracoscopic surgery (VATS), yielding mixed results. Falchuk et al. [9] attempted tetracycline-induced pleurodesis via tube thoracostomy in 2 patients with recurrent hepatic hydrothorax. One patient had no recurrent pleural effusion at 6 months of follow-up; however, the other patient died of a variceal hemorrhage 3 weeks following pleurodesis. Runyon et al. [10] reported lifethreatening massive protein and electrolyte depletion in 2 patients with hepatic hydrothorax following chest tube placement. In a study by Milanez de Campos et al. [11], pleurodesis was performed using talc insufflation via thoracoscopy (conventional or VATS) in 18 patients with refractory hepatic hydrothorax (21 procedures). Talc insufflation alone was effective in $10 / 21$ procedures (47.6\%), while talc insufflation associated with diaphragmatic defect suturing was successful in 3/5 (60\%). However, procedure-related morbidity was high, as empyema ( 2 patients), pneumonia (1 patient), persistent air leak (2 patients), or milder side effects (mild fever in 3 , chest pain in 2) occurred in a significant proportion of subjects. $\mathrm{Pa}$ tients, in whom diaphragmatic defects were not identified, experienced a more extensive period of drainage via chest tube (33\%), with ensuing electrolyte imbalance and albumin depletion. Four recurrences were retreated (19.1\%), but only 1 remained recurrence-free at 3 months of follow-up. Ferrante et al. [12] reported the outcomes of 15 patients who underwent VATS for refractory hepatic hydrothorax. Successful control of hydrothorax was achieved and maintained in $53 \%$ of patients after 1 VATS procedure with talc pleurodesis, and in $73 \%$ after 2 such procedures. Complications included mild fever and leukocytosis in $8 / 15$ patients (53\%), pleurocutaneous fistula in 3 , and empyema in 2.

Surgical repair of diaphragmatic leaks has been associated with the resolution of hepatic hydrothorax in cirrhotic patients, with a decreased risk of recurrence. As previously mentioned, Milanez de Campos et al. [11] were able to identify and suture diaphragmatic defects in 5/14 patients undergoing VATS. However, 1 patient had significant drainage for a prolonged period ( $>30$ days) followed by development of empyema, and another patient died 18 days after the procedure, secondary to pneumonia and liver failure. A similar experience was described by Mouroux et al. [13], where $6 / 8$ patients with refractory hepatic hydrothorax were found to have diaphragmatic defects on VATS. Diaphragmatic repair was undertaken via sutures or biologic glue followed by talc pleurodesis.
None of the 6 patients had recurrent pleural effusion despite the recurrence of ascites.

A novel therapeutic strategy for refractory hepatic hydrothorax has recently been described in a small case series. Five patients underwent thoracoscopically guided combined mechanical pleural abrasion and talc pleurodesis, in addition to intraperitoneal drain insertion. The latter prevented reaccumulation and translocation of ascites into the pleural cavity, therefore allowing parietal and visceral pleural inflammation and adhesion to proceed without interference of recurrent fluid. Four patients did not develop recurrent hepatic hydrothorax. One patient died 132 days following the procedure, secondary to complications of spontaneous bacterial peritonitis; the other 4 survived to liver transplantation. It is worth noting, however, that such a procedure is quite invasive. In fact, significant intraoperative bleeding in 1 patient with coagulation disorders secondary to liver cirrhosis was observed, requiring resuscitation. In addition, 4 of 5 patients had pleural or peritoneal fluid infections while drainage tubes were in place. However, all infections evolved favorably following antibiotic treatment [14].

Recently developed tunneled pleurocutaneous catheters seem promising with regard to the management of MPE. In a recent study, 222/231 (96.1\%) patients with MPE reported complete or partial symptom improvement following catheter insertion. Long-lasting spontaneous pleurodesis was achieved in $43.9 \%$. Catheters were in place for a median time of 56 days, and complication rates were acceptable, with empyema developing in only $3.2 \%$ of cases, and symptomatic loculation of fluid in $8.4 \%$ [1]. Curiously, few studies have explored the safety and efficacy of tunneled pleural catheters for refractory benign pleural effusions, and more specifically, transudative hepatic hydrothorax in patients with advanced cirrhotic liver disease. Murthy et al. [15] reported the use of PleurX catheters in 58 patients with complicated pleural effusions, 11 of whom had benign underlying conditions ( 3 patients with congestive heart failure, 2 with hepatic cirrhosis, and 6 with other benign disorders). Among 15 catheters placed in those patients with benign complicated pleural effusion, 6 were removed without recurrent effusion. However, more detailed analysis of safety and efficacy data was not specifically reported for this subcategory of patients. A recent case series described the use of PleurX catheters in 5 patients with refractory congestive heart failure-associated pleural effusion. The catheters remained in place for a period of 1-15 months. In 3 patients, the PleurX catheter was removed due to secondary sequelae. In 1 patient, the catheter stopped functioning 4 months following inser- 
tion. Two patients developed empyema 5 and 15 months after catheter placement; 1 of these patients had a favorable course with catheter removal, therapeutic thoracentesis and antibiotics, while the other patient - a 92-year-old lady - developed the full sepsis syndrome with multiorgan failure and death. Two patients had no catheter-induced complications at 1 and 1.5 months of follow-up. None of the patients developed obvious protein deficiency or electrolyte abnormalities related to prolonged drainage of pleural fluid [16]. In this study, the occurrence of spontaneous pleural symphysis in about half of the cases treated with an indwelling pleural catheter might prevent protein depletion. On the other hand, the amount of pleural fluid drained with a conventional large thoracic tube would probably provoke more protein and electrolyte depletion than the small-bore indwelling pleural catheter.

This case suggests that the PleurX pleural catheter can be a safe and effective noninvasive tool for the management of refractory hepatic hydrothorax. Major key advantages over previous approaches include its easy and simple insertion technique in an outpatient setting, without sedation or general anesthesia, its cost-effectiveness, and low complication rates. Concerns with regard to infectious complications (especially empyema) are justified. Use of indwelling pleural catheters in benign conditions may even be associated with higher rates of infectious adverse effects, as such catheters will remain in place for longer than is usual for cancer patients. Close follow-up and surveillance are, therefore, warranted. Although protein depletion and electrolyte abnormalities have previously been reported in patients undergoing standard tube thoracostomy drainage for hepatic hydrothorax [9], such complications were not seen in our patient despite a 6-month drainage period.

This case illustrates the novel use of tunneled pleural catheters for hepatic hydrothorax in patients with advanced cirrhosis. Although this seems promising, more data are needed to better analyze the safety and efficacy of pleural catheters for such indications.

\section{References}

1 Tremblay A, Michaud G: Single-center experience with 250 tunnelled pleural catheter insertions for malignant pleural effusion. Chest 2006;129:362-368

$\checkmark 2$ Jantz MA, Antony VB: Pathophysiology of the pleura. Respiration 2008;75:121-133.

$\checkmark 3$ Chen A: Massive hydrothorax complicating liver cirrhosis as a result of a diaphragmatic defect. Respiration 1988;54:206-207.

4 Garcia N, Mihas AA: Hepatic hydrothorax: pathophysiology, diagnosis and management. J Clin Gastroenterol 2004;38:52-58.

5 Singh C, Sager JS: Pulmonary complications of cirrhosis. Med Clin North Am 2009;93: 871-883.

-6 Gordon FD, Anastopoulos HT, Crenshaw W, Gilchrist B, McEniff N, Falchuk KR, LoCicero J 3rd, Lewis WD, Jenkins RL, Trey C: The successful treatment of symptomatic, refractory hepatic hydrothorax with transjugular intrahepatic portosystemic shunt. Hepatology 1997;25:1366-1369.
-7 Strauss RM, Martin LG, Kaufman SL, Boyer TD: Transjugular intrahepatic portal systemic shunt for the management of symptomatic cirrhotic hydrothorax. Thorax 1994 89:1520-1522.

-8 Spencer EB, Cohen DT, Darcy MD: Safety and efficacy of transjugular intrahepatic portosystemic shunt creation for the treatment of hepatic hydrothorax. J Vasc Intery Radiol 2002;13:385-390.

$\checkmark 9$ Falchuk KR, Jacoby I, Colucci WS, et al: Tetracycline-induced pleural symphysis for recurrent hydrothorax complicating cirrhosis: a new approach to treatment. Gastroenterology 1977;72:319-321.

10 Runyon BA, Greenblatt M, Ring MHC: Hepatic hydrothorax is a relative contraindication to chest tube insertion. Am J Gastroenterol 1986;81:566-567.

11 Milanez de Campos JR, Filho LO, de Campos Werebe E, Sette H Jr, Fernandez A, Filomeno LT, Jatene FB: Thoracoscopy and talc poudrage in the management of hepatic hydrothorax. Chest 2000;118:13-17.
12 Ferrante D, Arguedas MR, Cerfolio RJ, Collins BG, van Leeuwen DJ: Video-assisted thoracoscopic surgery with talc pleurodesis in the management of symptomatic hepatic hydrothorax. Am J Gastroenterol 2002;97: 3172-3175.

13 Mouroux J, Perrin C, Venissac N, Blaive B, Richelme H: Management of pleural effusion of cirrhotic origin. Chest 1996;109: 1093-1096.

-14 Northup PG, Harmon RC, Pruett TL, Schenk WG 3rd, Daniel TM, Berg CL: Mechanical pleurodesis aided by peritoneal drainage: procedure for hepatic hydrothorax. Ann Thorac Surg 2009;87:245-250.

15 Murthy SC, Okereke I, Mason DP, Rice TW: A simple solution for complicated pleural effusions. J Thorac Oncol 2006;1:697-700.

16 Herlihy JP, Loyalka P, Gnananandh J, Gregoric ID, Dahlberg CGW, Kar B, Delgado RM 3rd: PleurX ${ }^{\circledR}$ catheter for the management of refractory pleural effusions in congestive heart failure. Tex Heart Inst J 2009;36:38-43. 